\title{
Successful Surgical Abdominal Aortic Debranching Preceding Stent Graft Implantation: A Case Report
}

\author{
Magdalena L. Laux ${ }^{1}$ Michael Erb ${ }^{1} \quad$ Frank Hoelschermann ${ }^{2}$ Johannes M. Albes ${ }^{1}$ \\ ${ }^{1}$ Department of Cardiovascular Surgery, Brandenburg Heart Center, \\ University Hospital, Brandenburg Medical School Theodor Fontane, \\ Bernau, Germany \\ 2 Department of Cardiology, Brandenburg Heart Center, Bernau, Germany \\ Address for correspondence Magdalena L. Laux, MD, Department \\ of Cardiovascular Surgery, Brandenburg Heart Center, University \\ Hospital, Brandenburg Medical School Theodor Fontane, \\ Ladeburgerstr 17, 16321 Bernau, Germany \\ (e-mail:m.laux@immanuel.de).
}

Thorac Cardiovasc Surg Rep 2018;7:e24-e26.
Abstract
Background Acute endovascular aneurysm repair with stent grafts (thoracic endo- vascular aortic repair [TEVAR]) is safe and feasible.
Keywords
- TEVAR
- abdominal debranching
- celiac trunk
- perforated aortic aneurysm Case Description A 64-year-old female presented with a perforated aortic aneurysm of the thoracic descending aorta. Primary TEVAR resulted in good management of the perforation but a type Ib endoleakage remained postoperatively. To place another stent, abdominal debranching with saphenous vein bypass to the celiac trunk was required. In the same session, another endograft was inserted successfully.
Conclusion Abdominal debranching is a safe alternative to open aortic repair in acute thoracic and abdominal aneurysms, instead of waiting for a custom-made device.

\section{Introduction}

The incidence of rupture in descending thoracic aortic aneurysms is described as 5 per 100,000 , with a mortality rate of 97 to $100 \%{ }^{1}$ The predominant risk factor is hypertension. ${ }^{1}$ Treatment with thoracic endovascular aortic repair (TEVAR) has demonstrated a lower morbidity and mortality than conventional surgery. ${ }^{2}$ Aortic anatomy is sometimes a limitation for TEVAR but new options have become available in the last years. Endoleaks, however, present an unpredictable problem. Fenestrated or branched prostheses are an option, have to be custom-made, and are not helpful in an acute or subacute setting. Snorkels and chimneys can solve this problem but tend to have type I endoleaks, and a slightly higher complication rate than fenestrated endovascular aneurysm repair (EVAR). ${ }^{3}$ As an alternative, overstenting of major vessels is a good solution provided that debranching is performed before or during the procedure.

\section{Case Description}

A 64-year-old female with a history of hypertension, hypercholesterinemia, and obesity presented with back pain and dyspnea. Earlier external diagnosis included spinal magnetic resonance imaging showing a descending thoracoabdominal aortic aneurysm. A computed tomography (CT) led to the diagnosis of a covered ventral aortic perforation of the descending thoracic aorta approximately $5 \mathrm{~cm}$ above the diaphragm (-Fig. 1).

Taking the possible landing zones right below the left subclavian artery and cranially above the celiac trunk into account, we placed a thoracic stent graft (Bolton Medical Relay NBS Thoracic Stentgraft $36 \times 199 \mathrm{~mm}$, Sunrise, Florida, United States) into the descending aorta. The stent graft was then extended with a second graft (Bolton Medical Relay NBS $38 \times 154 \mathrm{~mm}$ ). A type Ib endoleak was initially treated with balloon dilatation of the second prosthesis. Back pain receded. Postinterventional CT scan showed a small endoleak initially understood as type II (-Fig. 2).

Within 1 month the patient was hospitalized for recurrent pain. The endoleak now presented as severe type Ib. Sealing of the endoleak by extension with another short graft failed due to shortening of the stent during release and therefore missing its optimal position just above the celiac trunk by $5 \mathrm{~mm}$. Thus, another extension was necessary. In a hybrid procedure, a venous bypass from the caudal abdominal aorta to the celiac received

January 2, 2018 accepted after revision May 2, 2018
DOI https://doi.org/

$10.1055 / \mathrm{s}-0038-1660834$. ISSN 2194-7635. (c) 2018 Georg Thieme Verlag KG Stuttgart · New York
License terms

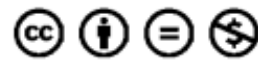



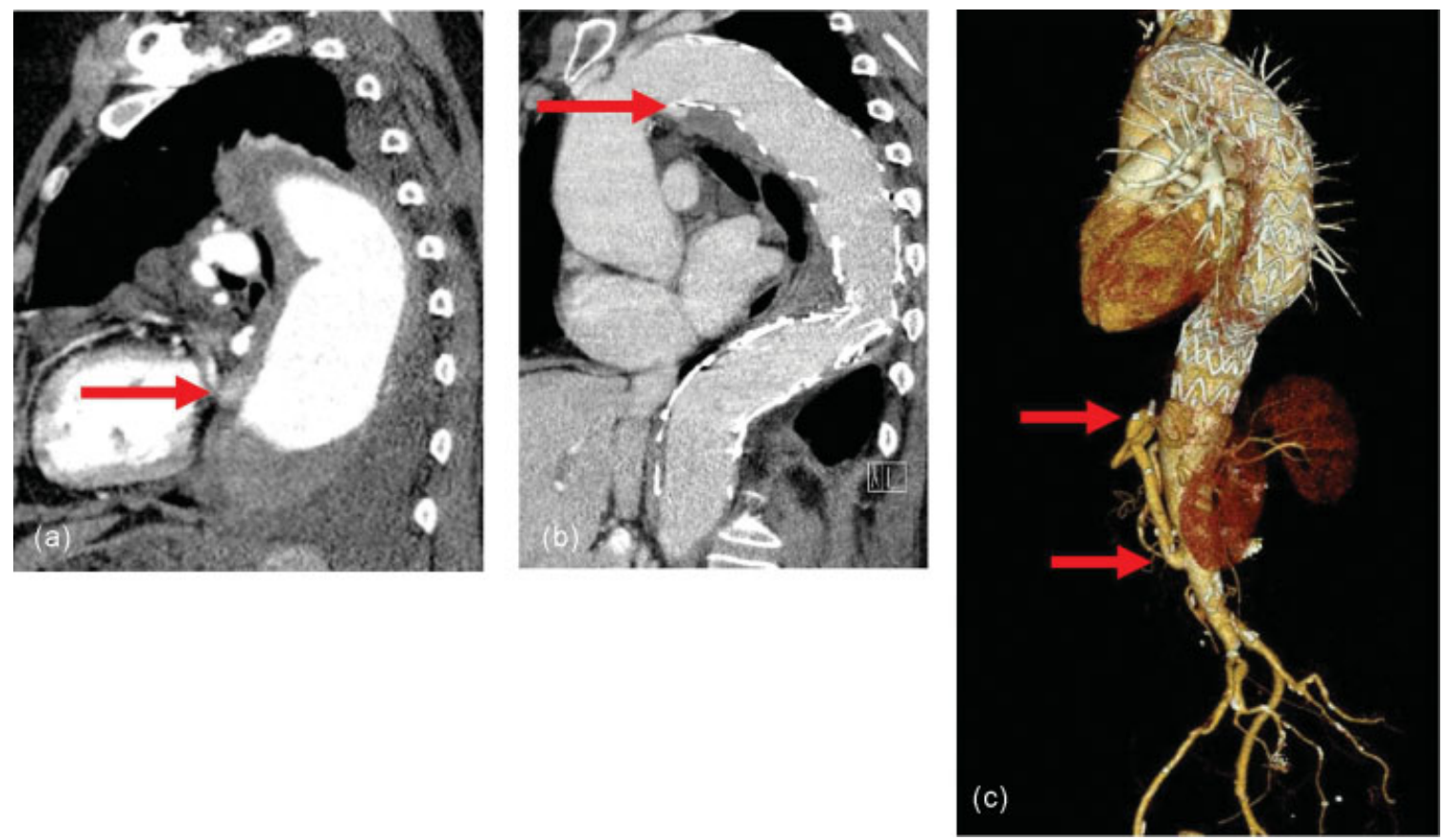

Fig. 1 (a) Initial computed tomography (CT) scan showing main part of the aneurysm in descending aorta, arrow points to perforation. (b) Aneurysm of the ascending aorta after 1 year. Arrow points to localized dilatation. (c) Result: Outer diameter of the aneurysm started to recede. Arrows show proximal and distal anastomosis of the celiac trunk bypass.

trunk was placed via median laparotomy followed by implantation of a fourth stent introduced via the abdominal aorta in the same session excluding the celiac trunk as expected (Bolton Medical Relay Plus $42 \times 105 \times 42 \mathrm{~mm}$ ). A peroneal palsy of the right foot was noted but decreased over time. The patient was discharged 2 weeks after the procedure, with planned follow-up at 3, 9, and 12 months and annual controls thereafter.

After 1 year, an aneurysm of the ascending aorta showed an increase in diameter of $1 \mathrm{~cm}$ reaching $5.6 \mathrm{~cm}$ in total extending to the landing zone of the first TEVAR ( - Fig. 1 ). We performed supracoronary replacement of the ascending aorta and aortic arch with a hybrid prosthesis (Jotec, Evita Open, $36 \mathrm{~mm}$, Jotec, Hechingen, Germany) under circulatory arrest connecting the stented part of this graft with the TEVAR. After an uneventful postoperative course the patient was discharged home.

\section{Discussion}

We describe a case of overstenting the celiac trunk with a TEVAR after debranching in a large thoracoabdominal aneurysm with a contained rupture. Adequate and close follow-up in TEVAR is necessary to detect further problems. Positioning of a TEVAR is always delicate owing to potential impairment of major arterial branches. ${ }^{4}$ Particularly in patients with acute symptoms and a complicated anatomy abdominal debranching is a safe option and offers an avenue out of the dilemma of a prolonged and thus potentially risky waiting time for a custom-made prosthesis with fenestrations and/or chimneys. ${ }^{5,6}$ In case of acute rupture, the mortality for EVAR is described as $40 \%$ versus $62.5 \%$ in surgically treated patients. ${ }^{7}$ There is no long-term data about mortality and endoleaks in snorkels and chimneys. Patency of visceral grafts is described as $97 \%$ after 19.3 months and 30-day mortality is 0 to $34 \%$ in the literature. ${ }^{8-10}$ We chose a hybrid
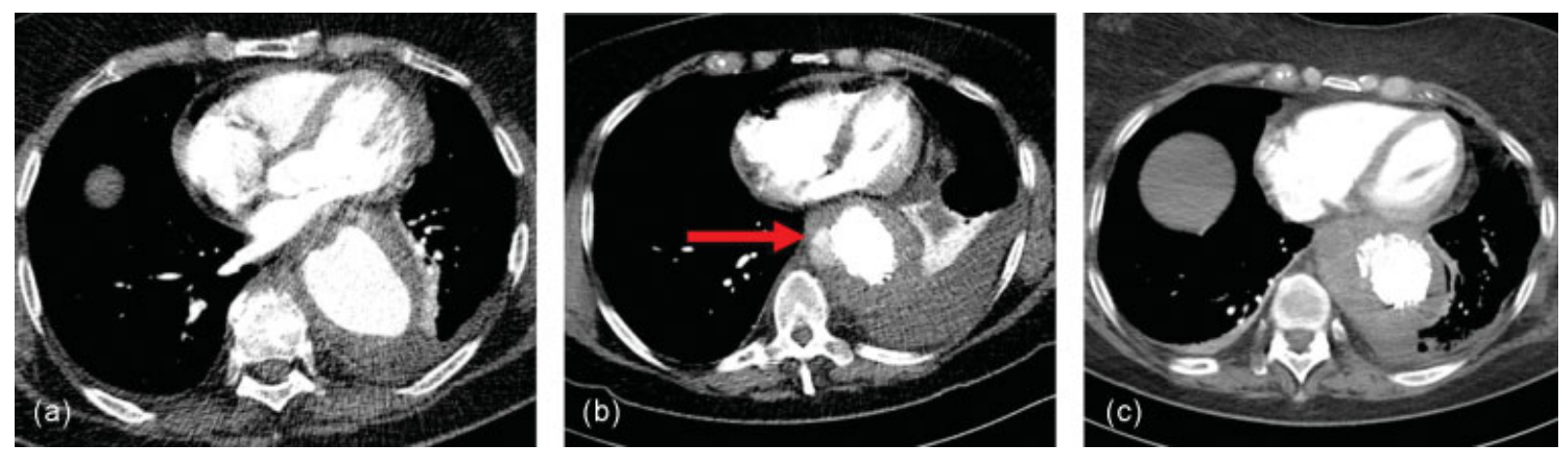

Fig. 2 (a) Before procedure. (b) After the first procedure. Arrow shows endoleak. (c) Final result after three procedures and aortoceliac bypass. No enhancement of contrast medium. Thrombus is solid, total exclusion of aneurysm. 
procedure in this young patient, expecting it to be the option with the best long-term outcome with no endoleaks. ${ }^{3}$ While available evidence is mostly anecdotal, the favorable outcome of our patient with abdominal bypass and TEVAR occluding the celiac trunk for treatment of a persisting endoleak is encouraging.

\section{Note \\ The article was presented in annual meeting, DGTHG Leipzig, Germany, February 13, 2017.}

\section{References}

1 Johansson G, Markström U, Swedenborg J. Ruptured thoracic aortic aneurysms: a study of incidence and mortality rates. J Vasc Surg 1995;21(06):985-988

2 Jackson RS, Chang DC, Freischlag JA. Comparison of long-term survival after open vs endovascular repair of intact abdominal aortic aneurysm among Medicare beneficiaries. JAMA 2012;307 (15):1621-1628

3 Katsargyris A, Oikonomou K, Klonaris C, Töpel I, Verhoeven EL. Comparison of outcomes with open, fenestrated, and chimney graft repair of juxtarenal aneurysms: are we ready for a paradigm shift? J Endovasc Ther 2013;20(02):159-169
4 Czerny M, Weigang E, Sodeck G, et al. Targeting landing zone 0 by total arch rerouting and TEVAR: midterm results of a transcontinental registry. Ann Thorac Surg 2012;94(01):84-89

5 Shijo T, Kuratani T, Shirakawa Y, et al. The assessment of collateral communication after hybrid repair for Crawford extent II thoracoabdominal aortic aneurysms. Eur J Cardiothorac Surg 2015;48 (06):960-967, discussion 967

6 Hughes GC, Andersen ND, Hanna JM, McCann RL. Thoracoabdominal aortic aneurysm: hybrid repair outcomes. Ann Cardiothorac Surg 2012;1(03):311-319

7 Dalainas I, Nano G, Bianchi P, et al. Endovascular techniques for the treatment of ruptured abdominal aortic aneurysms: 7-year intention-to-treat results. World J Surg 2006;30(10):1809-1814, discussion 1815-1816

8 Hughes GC, Barfield ME, Shah AA, et al. Staged total abdominal debranching and thoracic endovascular aortic repair for thoracoabdominal aneurysm. J Vasc Surg 2012;56(03):621-629

9 Damrauer SM, Fairman RM. Visceral debranching for the treatment of thoracoabdominal aortic aneurysms: based on a presentation at the 2013 VEITH Symposium, November 19-23, 2013 (New York, NY, USA). Aorta (Stamford) 2015;3(02):67-74

10 Fukui D, Wada Y, Komatsu K, et al. Innovative application of available stent grafts in Japan in aortic aneurysm treatmentsignificance of innovative debranching and chimney method and coil embolization procedure. Ann Vasc Dis 2013;6(03): 601-611 